\title{
Trends and patterns in the use of computed tomography in children and young adults in Catalonia - results from the EPI-CT study
}

\author{
Magda Bosch de Basea ${ }^{1,2,3}$ • Jane A. Salotti ${ }^{4}$. Mark S. Pearce ${ }^{4}$. Jordi Muchart ${ }^{5}$. \\ Luis Riera $^{6}$ • Ignasi Barber ${ }^{7}$ - Salvador Pedraza ${ }^{8,9,10}$ • Marina Pardina ${ }^{11}$. \\ Antoni Capdevila ${ }^{12}$ - Ana Espinosa ${ }^{1,2,3,13}$ - Elisabeth Cardis ${ }^{1,2,3}$
}

Received: 17 November 2014 / Revised: 26 May 2015 / Accepted: 9 July 2015 / Published online: 15 August 2015

(C) The Author(s) 2015. This article is published with open access at Springerlink.com

\begin{abstract}
Background Although there are undeniable diagnostic benefits of CT scanning, its increasing use in paediatric radiology has become a topic of concern regarding patient radioprotection.

Objective To assess the rate of CT scanning in Catalonia, Spain, among patients younger than 21 years old at the scan time.

Materials and methods This is a sub-study of a larger international cohort study (EPI-CT, the International pediatric CT scan study). Data were retrieved from the radiological information systems (RIS) of eight hospitals in Catalonia since the implementation of digital registration (between 1991 and 2010) until 2013.

Results The absolute number of CT scans annually increased 4.5\% between 1991 and 2013, which was less accentuated when RIS was implemented in most hospitals. Because the population attending the hospitals also increased, however, the rate of scanned patients changed little ( 8.3 to 9.4 per 1,000 population). The proportions of patients with more than one CT and more than three CTs showed a 1.51- and 2.7-fold increase, respectively, over the 23 years.
\end{abstract}

Magda Bosch de Basea

magda.boschdebasea@gmail.com

1 Centre for Research in Environmental Epidemiology (CREAL), Dr. Aiguader, 88, 08003 Barcelona, Spain

2 Universitat Pompeu Fabra (UPF), Barcelona, Spain

3 CIBER Epidemiología y Salud Pública (CIBERESP), Barcelona, Spain

4 Institute of Health \& Society, Newcastle University, Sir James Spence Institute of Child Health, Royal Victoria Infirmary, Newcastle upon Tyne, UK

5 Hospital Sant Joan de Déu Barcelona, Barcelona, Spain

6 Corporació Sanitària Parc Taulí, Sabadell, Spain
Conclusion Gradual increases in numbers of examinations and scanned patients were observed in Catalonia, potentially explained by new $\mathrm{CT}$ scanning indications and increases in the availability of scanners, the number of scans per patient and the size of the attended population.

Keywords Adolescents · Children · Cohortstudy · Computed tomography $\cdot$ Epidemiology $\cdot$ Trends

\section{Introduction}

Computed tomographic scanning is an extremely informative diagnostic technique, with a wide range of clinical applications. Because of the highly reduced imaging time compared to other techniques such as MRI, CT scanning suits all age groups, including children, without the need for anaesthesia or sedation. Since the introduction of the first CT unit in 1970, both the use of CT scans and the knowledge base and concerns

7 Hospital Universitari Vall d'Hebron, Barcelona, Spain

8 Institut de Diagnòstic per la Imatge (IDI), Hospital Universitari de Girona Doctor Josep Trueta, Girona, Spain

9 Institut d'Investigació Biomèdica de Girona Dr. Josep Trueta (IDIBGI),

Girona, Spain

10 Universitat de Girona, Girona, Spain

11 Hospital Universitari Arnau de Vilanova, Lleida, Spain

12 Hospital de la Santa Creu i Sant Pau, Barcelona, Spain

13 Hospital del Mar Medical Research Institute (IMIM), Barcelona, Spain 
about the potential deleterious health effects of ionising radiation exposure have grown in parallel. Over the last 20 years, CT scanner availability has rapidly increased in most European countries [1], including Spain, where the number of CT scan units per 100,000 population nearly doubled between 1996 and 2011 [2].

In Spain, almost 4 million scans were performed in 2011, representing a rate of $85 \mathrm{CT}$ scans per 1,000 people [2], whereas in Greece and the United States, countries with the highest rates of CT scans, the rate was three times higher (320.4 per 1,000 in Greece and 273.8 per 1,000 in the United States) [1].

Although CT scans comprise a small proportion of all diagnostic radiologic procedures, in countries like the United States they contribute nearly half the population's collective radiation dose from all medical X-ray examinations [3] and in the United Kingdom they account for up to $68 \%$ of the population's collective radiation dose [4]. In the U.S. more than 70 million CT scans are performed every year [5], whereas in the UK it surpasses the 5 million CT scans per year, with an increasing annual rate of $10 \%$ [6]. Overall, in Europe CT scanning is a critical source of ionising radiation exposure and its use is expected to increase in the next years with the widespread application of modern multi-slice techniques [7].

Predictions of the potential health impact of CT scanning [8] using a linear-no-threshold dose-response model (which postulates that the risk is linearly related to dose, with no safety threshold) have been controversial in the radiologic community. Some scientists have postulated that $\mathrm{CT}$ radiation doses are too low to produce any health effect [9]. However, recent direct assessment of the health effects of paediatric CT scanning through observational studies suggests an increased risk of leukaemia and brain tumours related to multiple CT scan exposure in children and adolescents [10,11]. Exposure in childhood is known to entail a higher risk of radiationinduced health effects than exposure later in life because childhood exposure is linked to greater cell division in growing and developing tissues and a longer life expectancy for diseases to develop $[9,12,13]$. Therefore the potential adverse health effects of paediatric CT have become an important topic of concern in radiologic protection [13]. Indeed, prediction models suggest that up to $2 \%$ of all future neoplasms in the general population of the United States could be attributable to CT scanning [14]. Even though for an individual child, the health risks from one CT scan are likely to be small and the individual benefit may outweigh the risks, many children are likely to receive multiple scans. It is therefore important that paediatric CT scans are properly indicated and performed and that the resulting radiation doses are closely monitored.

Information on the intensity and patterns of use of diagnostic radiology techniques in Spain is sparse. However, within Catalonia (a Northeast autonomous community of Spain) approximately 7.3 million diagnostic examinations were performed in 2007 on a population of 7.2 million people;
$83.8 \%$ of these diagnostic examinations involved ionising radiation and $7 \%(532,312)$ were CT scans [15]. Among the most common indications for $\mathrm{CT}$ imaging in children and young adults up to 20 years old are skull trauma and cephalgia with aggravated criteria, general abdominal problems when all other investigations (e.g., ultrasound) are inconclusive, and the diagnosis and follow-up of neoplasms [16].

Principles of radiation protection for medical exposures urge periodic evaluation of CT scan usage and substitution by other diagnostic techniques not involving ionising radiation, when possible, warranting accurate diagnosis without compromising the health of the patient. Monitoring of CT usage is important for updating the appropriateness criteria for ionising radiation imaging. The assessment of the use of CT scanning over time and characterisation of the patients referred for $\mathrm{CT}$ scanning (as part of a comprehensive strategy of evaluating individual risk) is a principal goal of the EPI-CT study.

The EPI-CT study (Epidemiological study to quantify risks for paediatric computerized tomography and to optimise doses) is a multinational cohort study of more than 1 million children, adolescents and young adults referred for CT scan supported by the European Union [17]. This paper assesses the patterns of use of CT in the Spanish autonomous region of Catalonia, one of the main contributors to the Spanish component of EPI-CT, among patients younger than 21 years old at the time that they had their first CT scan.

\section{Materials and methods}

\section{Study population}

Catalonia is the second largest autonomous community of Spain, with an estimated 2013 population of 7,553,650 people [18], including 1,599,195 (21.2\%) younger than 21 years [19], heterogeneously distributed over the $32,114 \mathrm{~km}^{2}$ that constitutes its four provinces (Barcelona, Tarragona, Lleida and Girona).

This study includes 8 public and autonomic-subsidised private hospitals out of 60 in Catalonia that provide health care free at the point of use for all children and young adults [20]. The eight hospitals providing data are among the larger medical centres performing radiology services to the majority of the paediatric and young adult patients in Catalonia (Hospital Sant Joan de Déu Barcelona, Hospital Universitari Vall d'Hebron, Corporació Sanitària Parc Taulí, Hospital Clínic de Barcelona, Hospital de la Santa Creu i Sant Pau, Hospital Universitari Joan XXIII de Tarragona, Hospital Universitari de Girona Dr. Josep Trueta and Hospital Universitari Arnau de Vilanova). These hospitals are part of the Catalan Health Care System (Xarxa Hospitalària d'Utilització PúblicaDepartament de Salut) and have been funded from autonomic general taxation since 2001 . It is reported that $24.3 \%$ of the Catalan adult population supplements their medical coverage 
through private health care, whereas only $17 \%$ of the population younger than 15 years uses private medical services [21]. No private hospitals were included in the study. Ethical approval was gained at each of the participating hospitals, as well as at the coordinating centre, Parc de Salut Mar, to retrospectively access these data.

Patients included in the study were accrued from the records of the radiology departments and had at least one CT scan before the age of 21 years. Completeness of data, defined as the collection of radiological information system (RIS) data from the start of CT scanning at the hospital, was only attained at one hospital. At the others, early records were not computerised and it was not possible to do so in the framework of the project.

\section{CT scan data}

The radiologic data were retrieved from the RIS (Radiology Information System) in each participating hospital since RIS implementation (which occurred heterogeneously between 1991 and 2010 among the hospitals) until December 2013. It is worth noting that by 2004 RIS was fully operating in all except for one small hospital, which in the following years only accounts for $1.6 \%$ of all scans in the study. RIS is a computerized system used to store, handle and circulate patient radiologic data. The RIS includes both patient identifying information and basic variables about the examination (including body part scanned and examination date and, in certain instances, indication for the CT scan and the referring hospital department). At one hospital, 2009-2011 data were extracted from the PACS (picture archiving and communication system) and SAP software (SAP SE, Walldorf, Germany) instead of RIS because the hospital discontinued RIS use and migrated to a more complex radiologic management system.

Data were obtained within the Spanish part of the multinational EPI-CT long-term cohort study.

Examination descriptions were grouped into six categories based on body part scanned, using Pearce et al.'s [22] adaptation of the categories defined by Mettler and collaborators [23]. This was done to ensure comparability of scan type groupings with the other EPI-CT participating countries. The categories were: head (including the neck), thorax, abdomen/pelvis, spine, extremities, and "several parts" (a composite of several scan locations in a single examination, e.g., head and thorax, thorax and abdomen, and thorax and pelvis). One of the biggest hospital contributors in terms of patients historically did not record the department ordering the $\mathrm{CT}$ examination, so in scans from that hospital the CT ordering service appears as "radiology and nuclear medicine," the default option.

\section{Statistical methods}

The use of CT imaging in the population younger than 21 years attending the participating hospitals was described using simple descriptive statistics. The data are described by gender and age at the time of the examination. For some analyses, children were categorized according to their age at the time of the scan in categories that ensure comparability with similar studies $(0,1$ to $<5,5$ to $<10,10$ to $<15,15$ to $<20$, and 20 to $<21$ years). The same reasoning was applied to the categorization of the number of CT scans $(1,2-5,6-10,11-$ $20,>20)$ for descriptive purposes. Chi-square test for homogeneity was used to assess the relationship between categorical variables. Both the number of CT scans and the number of scanned patients from 1991 to 2013 were modelled on the whole data set (including "hospital" as a cluster variable to take into account a random effect related to each hospital) as well as by hospital, fitting a linear regression model using a robust estimator of variance. For this, the outcome variable of these models (number of CT scans and number of scanned patients) was logged and transformed into a normally distributed variable. A generalised linear model with a logit link and binomial distribution was used to individually assess the change in the relative frequency of subjects with one $\mathrm{CT}$ scan per year over the study period. The same analysis was conducted to evaluate changes in the relative frequency of subjects with two, three and more than three CT scans per year. Each model was fit to the whole dataset (including hospital as a cluster variable) using a robust estimator of variance (Huber/ White sandwich estimator). A similar approach was used to model the variation on the frequency of body part scanned over time. To calculate the rates of CT scans, demographic data from the population served by the participating hospitals were obtained from the General Directorate of Planning and Research on Health (Direcció General de Planificació i Recerca en Salut); these data were available from the Catalan Health Department from 2005 to 2013 by 3-year time-bands. To estimate the population for the 2 years of unavailable data, a regression line was fitted using the official figures provided from 2005 till 2013. No population data were available for earlier periods. The Kruskal-Wallis test was used to compare the rate of CT scans per 1,000 population over time (2005-2013), which followed a non-parametrical distribution. All statistical analyses were performed using Stata (version 12.0; StataCorp, College Station, TX) and statistical significance was set at 0.05 .

\section{Results}

Between 1991 and 2013, 131,655 CT scans were performed on 74,437 children and adults younger than 21 years; 76,543 examinations $(58.1 \%)$ were performed on 42,238 males (56.7\%) and 55,112 (41.9\%) examinations on 32,199 females (43.3\%) (Table 1). Although the trend in the number of CT scans was heterogeneous among the hospitals, overall the number of CT scans increased by $4.5 \%$ ( $95 \%$ confidence 
Table 1 Characteristics of Catalan children and young adults referred for a CT scan by gender $\left(X^{2}, P<0.001\right.$ for all four categorical variables versus gender)

\begin{tabular}{|c|c|c|c|}
\hline Categorical variables & Totals & $\begin{array}{l}\text { Male } \\
n(\%)\end{array}$ & $\begin{array}{l}\text { Female } \\
n(\%)\end{array}$ \\
\hline Age at the time of the first scan (years) & 74,437 patients & 42,238 & 32,199 \\
\hline$<1$ & 6,796 & $3,876(9.2)$ & $2,920(9.1)$ \\
\hline 1 to $<5$ & 12,807 & $7,411(17.5)$ & $5,396(16.8)$ \\
\hline 5 to $<10$ & 12,911 & $7,138(16.9)$ & $5,773(17.9)$ \\
\hline 10 to $<15$ & 16,003 & $8,938(21.2)$ & $7,065(21.9)$ \\
\hline 15 to $<20$ & 21,807 & $12,649(29.9)$ & $9,158(28.4)$ \\
\hline 20 to $<21$ & 4,113 & $2,226(5.3)$ & $1,887(5.9)$ \\
\hline Age at the time of the scan (years) & 131,655 CT scans & & \\
\hline$<1$ & 10,555 & $6,084(7.9)$ & $4,471(8.1)$ \\
\hline 1 to $<5$ & 22,573 & $13,053(17.1)$ & $9,520(17.3)$ \\
\hline 5 to $<10$ & 23,220 & $13,107(17.1)$ & $10,113(18.3)$ \\
\hline 10 to $<15$ & 28,057 & $15,941(20.8)$ & $12,116(22.0)$ \\
\hline 15 to $<20$ & 39,822 & $24,018(31.4)$ & $15,804(28.7)$ \\
\hline 20 to $<21$ & 7,428 & $4,340(5.7)$ & $3,088(5.6)$ \\
\hline Body part scanned & 131,655 CT scans & & \\
\hline Head and neck & 79,885 & $46,983(61.4)$ & $32,902(59.7)$ \\
\hline Chest/thorax & 23,900 & $13,520(17.7)$ & $10,380(18.8)$ \\
\hline Abdomen/pelvis & 13,994 & $8,025(10.5)$ & $5,969(10.8)$ \\
\hline Spine & 5,849 & $3,456(4.5)$ & $2,393(4.3)$ \\
\hline Extremities & 4,894 & $2,748(3.6)$ & $2,146(3.9)$ \\
\hline Several parts & 1,338 & $740(1.0)$ & $598(1.1)$ \\
\hline Unknown & 1,795 & $1,071(1.4)$ & $724(1.3)$ \\
\hline Number of CT scans & 74,437 patients & & \\
\hline 1 & 52,498 & $29,178(69.1)$ & $23,320(72.4)$ \\
\hline $2-5$ & 18,797 & $11,144(26.4)$ & $7,653(23.8)$ \\
\hline $6-10$ & 2,322 & $1,437(3.4)$ & $885(2.7)$ \\
\hline $11-20$ & 728 & $435(1.0)$ & $293(0.9)$ \\
\hline$>20$ & 92 & $44(0.1)$ & $48(0.1)$ \\
\hline
\end{tabular}

interval [CI] 2.0-7.2\%) per year between 1991 and 2013. Increasing trends were seen in the number of CT scans in six of the eight participating hospitals, from the implementation of RIS in each hospital until the end of data collection in 2013 (Fig. 1); increases were statistically significant for the two biggest hospitals out of the eight participating centres. These two hospitals, which in 2013 performed 3,200 and 4,100 CT scans, respectively, in patients age 0-20, were among those where CT scan frequency showed a statistically significant anual growth rate of $8.1 \%$ and $5.6 \%$, respectively, over the study period (1991 to 2013).

The overall upward trend in number of CT scans per year declined in 2012 but seemed to recover by the end of the study period (2013). The increasing trend was less pronounced (3.6\% per year; 95\% CI 1.2-6.0\%) but was still statistically significant when considering the number of patients referred for CT scans (Fig. 1). By 2004 seven out of the eight participating hospitals had digitalised their radiology service data and there was a clear upward trend in absolute number of CT scans (Fig. 1) and in absolute number of patients (Fig. 1) over time, driven mainly by the increase in one of the biggest hospitals. The number of CT scanners per 100,000 population in Catalonia also increased during the period of 1996 to 2011 (Fig. 1). Although the starting point in terms of the number of patients and CT scans is similar for male and female patients, substantially more male patients received CT scans through the 23-year period (Table 1).

Median age at the time of the scan was 12.1 years old for male patients (interquartile range [IR]: 5.0-17.1) and 11.6 for female patients (IR: 4.9-16.7) (data not shown); 9.2\% of the patients in the study had their first CT scan during the first year of life. The age group that accounted for the largest number of CT scans was the 15 - to $<20$-years age group, with more than $30 \%$ of all the examinations performed in this age range. The overall distribution of CT scans by age-bands was very similar

Fig. 1 Trends in CT examinations from 1991 to 2013. a CT scans per year in children and young adults in Catalonia by hospital. b Number of children and young adults referred for CT imaging in Catalonia by hospital 

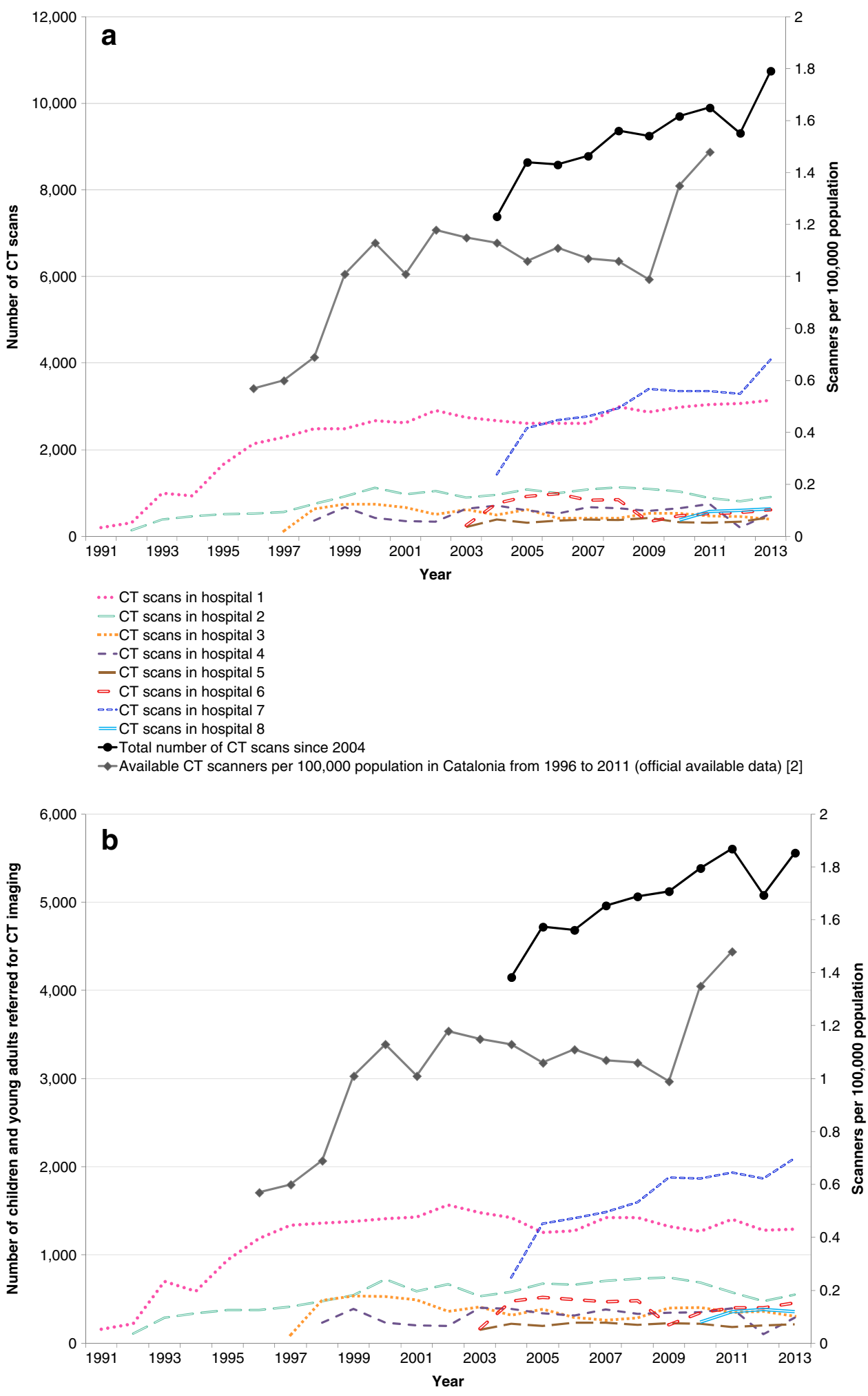

... Patients in hospital 1

- Patients in hospital 2

-...Patients in hospital 3

- -Patients in hospital 4

- Patients in hospital 5

- Patients in hospital 6

-. Patients in hospital 7

$=$ Patients in hospital 8

- Total number of scanned patients since 2004

$\rightarrow$ Available CT scanners per 100,000 population in Catalonia from 1996 to 2011 (official available data) [2] 
to the CT scan distribution in age-bands at the time of the first CT scan. Most patients, $70.5 \%$, received only one CT scan during the study period; $72.4 \%$ of all female patients had one CT scan and $23.8 \%$ had two to five CTs, while $69.1 \%$ of all male patients had one CT scan and $26.4 \%$ had two to five CTs (Table 1).

Within the participating hospitals, the number of CT scans performed in the same patient per year appeared to increase over time (Fig. 2). In 1991, 12\% of all the scanned patients received two $\mathrm{CT}$ scans, $1.2 \%$ received three $\mathrm{CT}$ scans and $1.2 \%$ received more than three CT scans. In 2013 at the end of data collection, $12.9 \%$ of all scanned patients received two CT scans, $4.2 \%$ received three CT scans and 5.1\% received more than three CT scans; the last category showed the greatest difference between the relative frequency in 1991 and 2013. The percentage of patients requiring only one CT scan showed a 0.6-fold decline between 1991 and 2013 $(P=0.076)$ (Fig. 2). In total, from 1991 to 2013 we observed
Fig. 2 Frequency of patients who received 1,2, 3 and more than 3 CT scans per year between 1991 and 2013

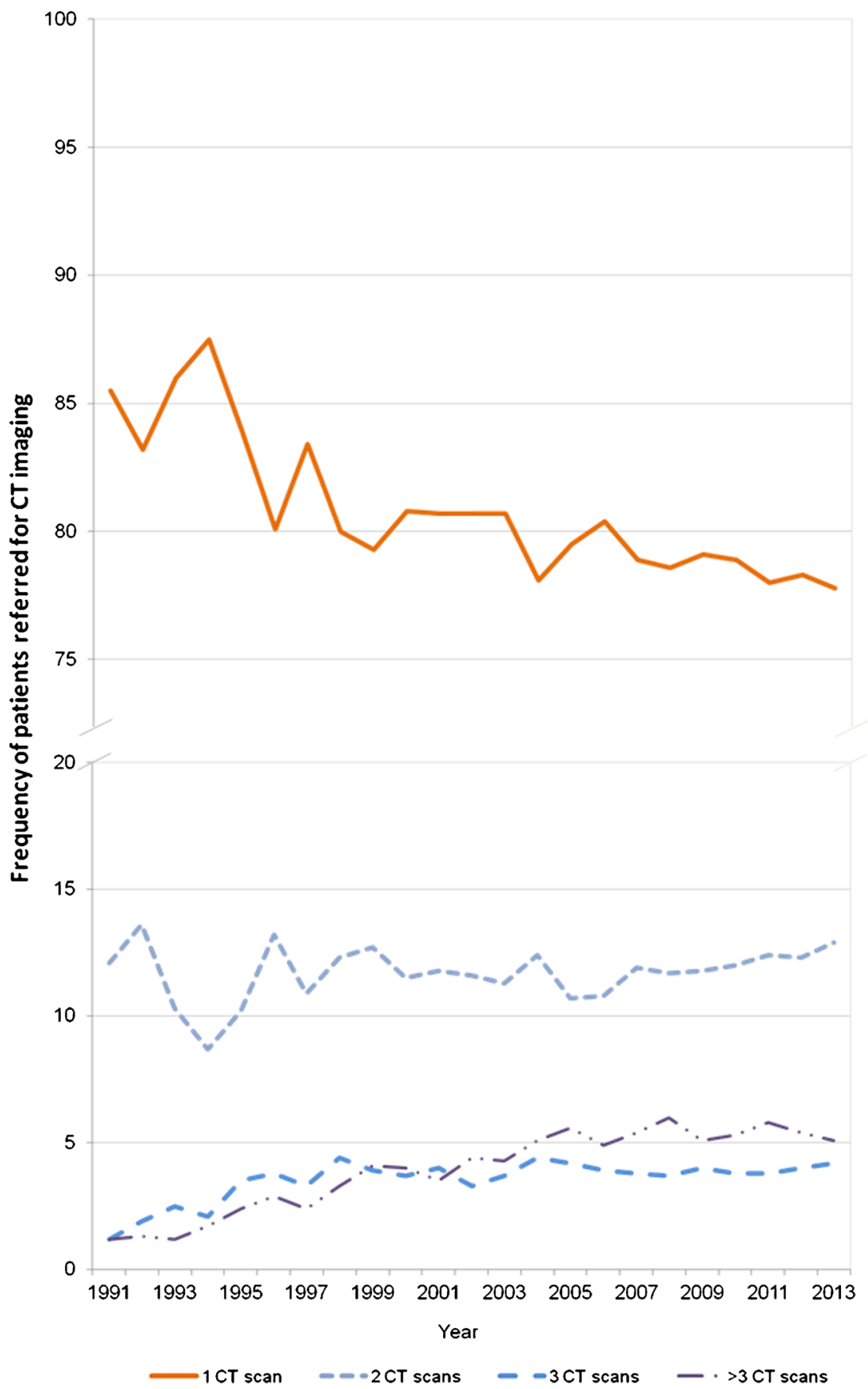


a non-statistically significant 1.5 -fold increase in the relative frequency of patients who received more than one CT scan per year $(P=0.076)$. During the same period the frequency of patients receiving more than three $\mathrm{CT}$ scans per year showed a 2.7 -fold increase $(P<0.001)$, although from 2004 onwards the trend is almost flat. When analysing by gender, similar trends were observed for male and female patients (data not shown), although comparatively during the total study period the frequency of female patients receiving one CT scan was higher than in males.

A total of 710 examination descriptions were identified from among 131,655 datasets in the study and were grouped into Mettler categories. In 101 examination records, the description of the body part scanned was missing, and 1,795 examination descriptions did not fit into any of the six Mettler categories. Three anatomical regions accounted for $90 \%$ of CT scans, with head/neck, thorax, and abdomen/pelvis representing $60.7 \%$, $18.2 \%$ and $10.6 \%$, respectively, of all CT scans. No differences were observed by gender (Table 1). The relative frequency of head/neck and abdomen/pelvis scans showed a 0.7-fold and 0.9fold decrease, respectively, over the 23-year period of the study, whereas the frequency of thorax examinations showed a 1.1fold increase (data not shown). When the heterogeneity among hospitals was taken into account no statistically significant trend was observed for the three most prevalent body parts scanned.

The distribution of CT scans by anatomical region varied depending on the age of the patient at the time of the diagnostic procedure. For patients younger than 10 years, approximately $66.5 \%$ of all CT scans performed were of the head/neck, with $21 \%$ of the thorax and $6.8 \%$ of the abdo$\mathrm{men} /$ pelvis. On the other hand, for patients older than 15 years, approximately $53.6 \%$ of CT scans were of the head/neck, $15.6 \%$ were of the thorax and $15.9 \%$ were of the abdomen/ pelvis (Table 2).

Information about the clinical department that requested the CT scan procedure was obtained directly from hospital RIS data for 111,995 CT scans ( $85.1 \%$ of all CT scans collected). Paediatrics departments requested $15.4 \%$ of the scans, followed by the surgery $(9.2 \%)$, neurosurgery and neurology $(8.6 \%)$, emergency $(7.1 \%)$ and oncology and haematological malignancies (5.2\%) (Table 3 ). There were statistically significant differences between referring specialities when comparing male with female patients (excluding "obstetrics and gynaecology" from the comparison) $(P<0.001)$. Approximately $12.2 \%$ of all CT scans performed in patients younger than 5 years were requested by neurosurgery and neurology, and this frequency decreased to $5.5 \%$ for the 20 -year-old patients. On the other hand, the emergency department requested $4.1 \%$ of all CT scans in those younger than 15 years and requested up to $13.1 \%$ of all CT scans performed in patients age 15 to $<20$ years. As might be expected, the frequency of the scans requested by paediatric departments decreased with the age of the patients. As explained in methods, in one of the biggest participating hospitals the "radiology and nuclear medicine" department was the default option when recording the department ordering the CT scan, which is not very informative of the CT scan distribution among medical departments in that larger hospital (Table 3).

When the number of CT scans and scanned patients per year was compared to the general referral population of similar age attended at the participating hospitals, there was a nonstatistically significant change from a rate of 8.3 scanned patients per 1,000 population in 2005 to 9.4 scanned patients/1,000 population in 2013 (Fig. 3). In terms of the number of CT scans per 1,000 population, the rate changed from $15.2 \mathrm{CT}$ scans in 2005 to 16.6 in 2012, with a subtle increase in 2013 to 18 CT scans per 1,000 population (not statistically significant). No information on the referral population was available for earlier years.

\section{Discussion}

Statistics on the use of CT in the National Spanish Health System have been provided by the Ministry of Health, Social Services and Equality since 1996 [2]. According to official data, the late 1990s witnessed the beginnings of an

Table 2 Body part scanned by age range for children and young adults in Catalonia from 1991 to $2013\left(X^{2}, P<0.001\right)$

\begin{tabular}{|c|c|c|c|c|c|c|}
\hline \multirow[b]{2}{*}{ Body part scanned } & \multicolumn{6}{|c|}{ Age at the time of the scan (years) } \\
\hline & $\begin{array}{l}<1 \\
n(\%)\end{array}$ & $\begin{array}{l}1 \text { to }<5 \\
n(\%)\end{array}$ & $\begin{array}{l}5 \text { to }<10 \\
n(\%)\end{array}$ & $\begin{array}{l}10 \text { to }<15 \\
n(\%)\end{array}$ & $\begin{array}{l}15 \text { to }<20 \\
n(\%)\end{array}$ & $\begin{array}{l}20 \text { to }<21 \\
n(\%)\end{array}$ \\
\hline Head/neck & $7,286(69.0 \%)$ & $15,023(66.6 \%)$ & $15,155(65.3 \%)$ & $17,154(61.1 \%)$ & $21,500(54.0 \%)$ & $3,767(50.7 \%)$ \\
\hline Thorax & $2,381(22.6 \%)$ & $4,876(21.6 \%)$ & $4,580(19.7 \%)$ & $4,722(16.8 \%)$ & $6,115(15.4 \%)$ & $1,226(16.5 \%)$ \\
\hline Abdomen/pelvis & $552(5.2 \%)$ & $1,548(6.9 \%)$ & $1,769(7.6 \%)$ & $2,583(9.2 \%)$ & $6,133(15.4 \%)$ & $1,409(19.0 \%)$ \\
\hline Spine & $136(1.3 \%)$ & $512(2.3 \%)$ & $707(3.0 \%)$ & $1,349(4.8 \%)$ & $2,675(6.7 \%)$ & $470(6.3 \%)$ \\
\hline Extremities & $71(0.7 \%)$ & $178(0.8 \%)$ & $594(2.6 \%)$ & $1,630(5.8 \%)$ & $2,146(5.4 \%)$ & $275(3.7 \%)$ \\
\hline Several parts & $67(0.6 \%)$ & $248(1.1 \%)$ & $194(0.8 \%)$ & $244(0.9 \%)$ & $463(1.2 \%)$ & $122(1.6 \%)$ \\
\hline Unknown & $62(0.6 \%)$ & $188(0.8 \%)$ & $221(1.0 \%)$ & $375(1.3 \%)$ & $790(2.0 \%)$ & $159(2.1 \%)$ \\
\hline Total & $10,555(8.0 \%)$ & $22,573(17.1 \%)$ & $23,220(17.6 \%)$ & $28,057(21.3 \%)$ & $39,822(30.2 \%)$ & $7,428(5.6 \%)$ \\
\hline
\end{tabular}




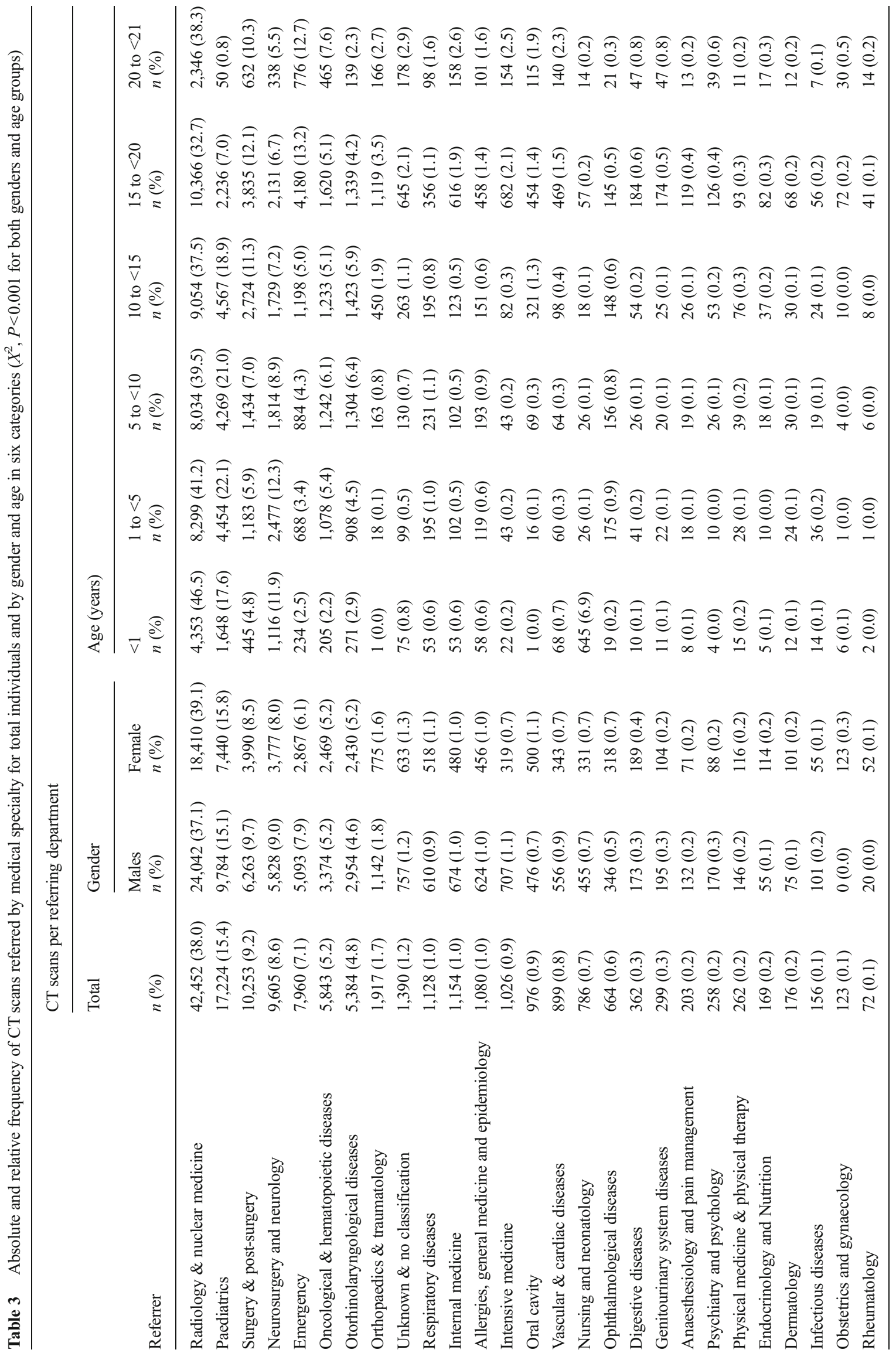


Fig. 3 Rate of children and young adults referred for $\mathrm{CT}$, and rate of CT scans per 1,000 people in the referral population (age 21 and younger) in the eight participating hospitals in Catalonia (2005-2013)

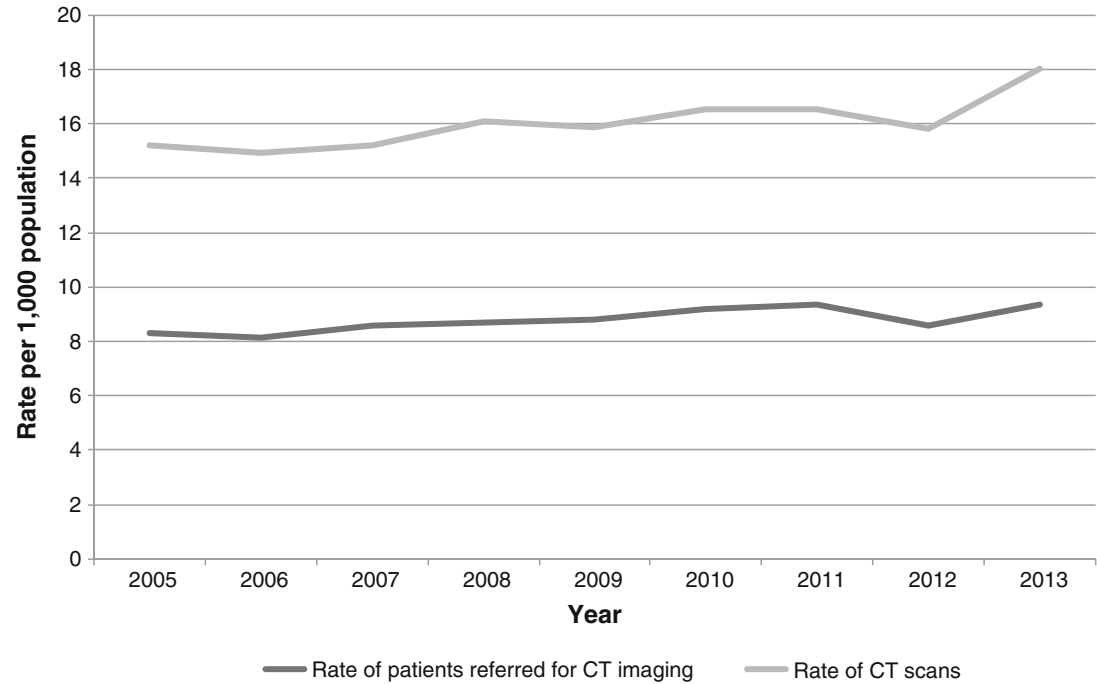

exponential use of CT imaging, with a $143 \%$ increase in the annual rate of CT scans (34.9 to $85.0 \mathrm{CT}$ scans per 1,000 population) from 1996 to 2011 in Spain, 2011 being the calendar year with the latest available information [2]. In Catalonia, based on our results, a sustained increase in the number of CT scans and a less pronounced increase in the number of scanned patients were observed during the 23year study period (1991-2013). The gradual digitalization of the radiology services among the participating hospitals, an increase in the number of scans per patient, an increase in the referral population attended at the participating hospitals, the greater number of CT scanners within the participating hospitals and the technical advances in $\mathrm{CT}$ equipment that allow implementation for new indications may explain the increase seen in the absolute number of CTs in the last two decades in these hospitals. It is important to emphasize that since 2004, when RIS was fully operating in all but one of the participating hospitals, an increase in the number of CT scans and scanned patients was evident though slightly less accentuated than in previous years. Therefore, the gradual RIS implementation in the hospitals may have affected the results in the number of CT scans and scanned patients in the early years, but not when most hospitals were routinely using RIS to manage and store their radiology, so we are confident that these results reliably reflect $\mathrm{CT}$ scan use.

The observed decrease in 2012 could be related to the increasing awareness of cancer risks following the publication of Pearce et al. [10], showing an increased risk of leukaemia and brain tumours in young people from CT scanning, which could have had an effect on the clinical practice of the radiologists at the participating hospitals. Additional data on CT scan frequency in the next years would be required to confirm this hypothesis. Most of the scanned patients received only one CT scan, although performing multiple CT scans in the same patient became increasingly common practice. Between 1991 and 2013, an increase in the frequency of patients receiving more than three $\mathrm{CT}$ scans per year was observed, suggesting a change in the clinical practice favouring the follow-up of diseases through CT scanning or a gradually wider use of CT scans for new indications. Although the current knowledge on enhanced radiation sensitivity of neonates and young infants is well known [14], a significant proportion of patients in the study had their first CT scans at very young ages ( $26.3 \%$ of the patients were younger than 5 years).

In Spain no information has been published on the trends and patterns of use of radiologic diagnostic procedures in children and young adults, hence the importance of describing the use of medium- to high-dose radiologic procedures in these patients. In the participating Catalan hospitals no increasing trend in the rate of scanned children and young adults and scans per 1,000 population was observed in the period 2005-2013, when official referral population figures were available. While the rate of CT scans and scanned patients per 1,000 population has not increased, there has been a considerable increase in the absolute number of CT scans and children and young adults referred for $\mathrm{CT}$ imaging in the 8year period for which official demographic data are available, which is closely followed by a proportional increase in the population of those younger than 21 years served by the participating hospitals. Over this 8-year period, the rate of young patients scanned per 1,000 in the referral population exceeded the doubling of the rate observed in Northern England in 2002 [24] and was similar to the rate described in Australia [25] for the 5 to $<15$ years age range in the same period.

The availability of CT scanners has increased rapidly in most major industrialized countries over the last two decades [1], which in most cases has translated into a swift augmentation of the number of CT scans performed yearly. In the whole of Spain, the availability of CT scanners doubled from 1996 to 2011 (available data period), from 0.9 to $1.6 \mathrm{CT}$ scanners per 100,000 population. In Catalonia, the number of CT scanners tripled in the same time period, from 0.6 to 1.5 scanners per 
100,000 population, suggesting potentially different intensities of use in this autonomous community [2]. The observed increase in scanner availability could respond to a growing demand of this imaging equipment since their gradual introduction in the Catalan hospitals. The escalating array of diagnostic and therapeutic CT scan indications, the nature of the services provided by the participating hospitals and the increasing attending population could have determined the adoption of this diagnostic technology at the hospitals and therefore the upward trend in available scanners.

A greater use of high-dose CT examinations such as head CT (including neck examinations), thorax, and abdomen (including pelvis examinations) was observed in Catalonia and similarly in the United States [26], United Kingdom [24], and the Netherlands [27] (although in the last, "extremities" imaging exceeded the frequency of "abdomen" imaging). Although there were differences in absolute and relative frequency of imaging among children and young adults, these three anatomical regions accounted for $76 \%$ of scans in the United States and 90.9\% in Northern England among CT scanning performed in children and young adults, suggesting similar uses in these two countries, as well as in Catalonia. The Netherlands study presented a closer distribution of frequency to that observed in the present study for head/neck and thorax imaging [27]. The potential decrease observed in the relative frequency of head/neck imaging over the study period could be attributed to a shift in the use of CT scans to other non-ionising diagnostic techniques. Regarding the decrease in the relative frequency of head CT scans, MRI has progressively become the gold standard for the investigation of cephalgia, especially in outpatient visits, providing wider detailed information than $\mathrm{CT}$. With regard to the decrease in the relative frequency of neck CTs, the technical improvements in ultrasound have allowed its use for the investigation and follow-up of neck disorders (such as lymphadenopathies, abscesses, tumours and branchial cleft cysts) and abdomen disorders (such as Crohn disease) that previously were commonly investigated using CT imaging. On the other hand, the relative frequency of thorax $\mathrm{CT}$ imaging seemed to increase, potentially explained by the non-existence of a diagnostic alternative beyond conventional radiology to investigate, e.g., lung parenchyma disorders.

The rates of the referring medical specialties in Catalonia compared to other countries suggest national variation in radiologic diagnostic practices; for comparison, in Denmark the most common referring specialty was orthopaedics, followed by paediatrics and then oncology, digestive system specialities, and surgery [28], whereas the majority of requests in Catalonia were from paediatrics, surgery, and neurosurgery and neurology specialties.

It is important to bear in mind that paediatric surgery does not exist as a medical specialty in most countries excepting Catalonia. Typically, in most paediatric hospitals different surgical specialties are encompassed under a wider paediatric surgery department, possibly explaining why surgery, is among the specialties ordering most of the CT scans.

The difference in the medical specialties ordering a CT scan for very young children compared to those ordering a $\mathrm{CT}$ for the 15 - to $<20$ years age group suggests different rates in specific pathologies and injuries. CTs in younger children could be related to congenital and neurodevelopmental abnormalities and also to a higher frequency of brain tumours typically seen in the youngest age groups, whereas the high rate of CT scanning in the emergency ward in adolescents and young adults could be related to a higher number of traumatic episodes, including road accidents, quarrels and suicides.

CT has been the cornerstone of imaging for more than 20 years, but orders for $\mathrm{CT}$ and its associated ionising radiation have to balance favourably with the diagnostic benefits. In the present study, $4.2 \%$ of patients received more than five CT scans before turning 21 years old and $1.2 \%$ received 10 or more $\mathrm{CT}$ scans, 41 being the maximum number of $\mathrm{CT}$ scans recorded in the same patient. In addition to the widely studied risks associated with the ionising radiation exposure of CT scans, it is important to take into account the economic burden associated with this procedure, especially in recession times where all public health expenditure across countries of the Organisation for Economic Co-operation and Development (OECD) have experienced cuts [1]. It is precisely this economic climate that, together with the increasing consciousness of the potential health effects of the procedure, may have played a role in modulating the high demand of these expensive medical technologies, but further analysis comparing countries with public and private health systems is needed to validate this.

\section{Conclusion}

This study showed the patterns of use of CT imaging in patients younger than 21 years in eight major medical centres treating paediatric and young adult patients in Catalonia during a 23-year study period. The observed gradual increase in examinations and patients referred for CT imaging may be related to an increase in the availability of CT scanners within the hospitals, an increase in the population attended in the participating hospitals, an increase in the number of scans per patient and new CT scanning indications. The initial findings that showed an upward trend in the use of CT scanning in the eight participating hospitals are in agreement with previous studies on paediatric CT scan use.

Acknowledgments The authors gratefully acknowledge the contribution of Jordi Figuerola and Álex Albert from CREAL, for the validation of the data and the thoughtful discussions.

Funding Supported in part by the Seventh Framework Programme from the European Community (Grant agreement no: 269912) and the Consejo de Seguridad Nuclear. 


\section{Conflicts of interest None}

Human participant protection The Ethics Committee of the Parc de Salut Mar and of the eight participating medical centres approved the study protocol.

Open Access This article is distributed under the terms of the Creative Commons Attribution 4.0 International License (http:// creativecommons.org/licenses/by/4.0/), which permits unrestricted use, distribution, and reproduction in any medium, provided you give appropriate credit to the original author(s) and the source, provide a link to the Creative Commons license, and indicate if changes were made.

\section{References}

1. OECD (2013) Medical technologies. In: Health at a Glance 2013: OECD indicators. http://www.oecd.org/health/health-systems/ health-at-a-glance.htm. Accessed 3 Oct 2014

2. Ministry of Health, Social Services and Equality (2014) [Key indicators of the NHS: INCLASNS - database]. http://www.msssi.gob. es/estadEstudios/estadisticas/sisInfSanSNS/inclasSNS_DB.htm. Accessed 8 Aug 2014

3. National Council on Radiation Protection and Measurements (2009) Ionizing radiation exposure of the population of the United States. NCRP, Bethesda, MD

4. Hart D, Wall BF, Hillier MC et al (2010) Frequency and collective dose for medical and dental X-ray examinations in the UK, 2008. National Radiological Protection Board, Oxfordshire

5. Brenner DJ (2010) Slowing the increase in the population dose resulting from CT scans. Radiat Res 174:809-815

6. NHS England Analytical Services (2014) NHS imaging and radiodiagnostic activity. United Kingdom

7. Regulla DF, Eder H (2005) Patient exposure in medical X-ray imaging in Europe. Radiat Prot Dosimetry 114:11-25

8. Brenner DJ, Elliston CD, Hall EJ et al (2001) Estimated risks of radiation-induced fatal cancer from pediatric CT. AJR Am J Radiol 176:289-296

9. Committee on the Biological Effects of Ionizing Radiation (2006) Health risks from exposure to low levels of ionizing radiation. BEIR VII report, phase II. US National Research Council, Washington DC

10. Pearce MS, Salotti JA, Little MP et al (2012) Radiation exposure from CT scans in childhood and subsequent risk of leukaemia and brain tumours: a retrospective cohort study. Lancet 380:499-505

11. Mathews JD, Forsythe AV, Brady Z et al (2013) Cancer risk in 680,000 people exposed to computed tomography scans in childhood or adolescence: data linkage study of 11 million Australians. BMJ 346:f2360

12. UNSCEAR (2010) Sources and effects of ionizing radiation. United Nations, Vienna
13. ICRP, Khong P-L, Ringertz $\mathrm{H}$ et al (2013) ICRP publication 121: radiological protection in paediatric diagnostic and interventional radiology. Ann ICRP 42:1-63

14. Brenner DJ, Hall EJ (2007) Computed tomography — an increasing source of radiation exposure. N Engl J Med 357:2277-2284

15. Ministry of Health. General Directorate of Planning and Evaluation (2008) Survey of diagnostic imaging in Catalonia. Quantitative survey. 2007 data. [Enquesta de diagnòstic per la imatge a Catalunya. Enquesta quantitativa. Dades de 2007]

16. American College of Radiology (2015) ACR appropriateness criteria. https://acsearch.acr.org/list. Accessed 31 March 2015

17. Bosch de Basea M, Pearce MS, Kesminiene A et al (2015) EPI-CT: Design, challenges and epidemiological methods of an international study on cancer risk after paediatric CT. J Radiol Prot 35 611-28

18. Statistical Institute of Catalonia (Idescat) Registration Office. Municipal census by sex. Official figures in Catalonia. [Padró municipal d'habitants per sexe. Xifres oficials Catalunya]. http://www. idescat.cat/territ/basicterr?TC $=8 \& \mathrm{~V} 3=669 \& \mathrm{~V} 4=446 \& \mathrm{ALLINFO}=$ TRUE\&PARENT $=1 \& \mathrm{~V} 0=3 \& \mathrm{~V} 1=0 \& \mathrm{CTX}=\mathrm{B} \& \mathrm{VN}=3$. Accessed 8 Aug 2014

19. Statistical Institute of Catalonia (Idescat) Registration Office. Population by sex and age per year. Catalonia 2013. [Població segons sexe i edat any a any. Catalunya Any 2013.] http://www. idescat.cat/territ/BasicTerr?TC $=5 \& \mathrm{~V} 0=3 \& \mathrm{~V} 1=0 \& \mathrm{~V} 3=669 \& \mathrm{~V} 4=$ $1180 \& A L L I N F O=T R U E \& P A R E N T=1 \& C T X=B$. Accessed 8 Aug 2014

20. Ministry of Health. General Directorate of Planning and Evaluation (2007) Strategic plan for pediatric care in primary health care. [Pla Estratègic d'Ordenació de l'Atenció de Pediatria a l'Atenció Primària]

21. Ministry of Health. General Directorate of Health Care Regulation (2013) Medical insurance entities of Catalonia. 2012. [Entitats d'assegurança sanitària lliure de Catalunya. 2012. Generalitat de Catalunya]

22. Pearce MS, Salotti JA, McHugh K et al (2012) Socio-economic variation in CT scanning in Northern England, 1990-2002. BMC Health Serv Res 12:24

23. Mettler FA Jr, Wiest PW, Locken JA et al (2000) CT scanning: patterns of use and dose. J Radiol Prot 20:353-359

24. Pearce MS, Salotti JA, McHugh K et al (2011) CT scans in young people in Northern England: trends and patterns 1993-2002. Pediatr Radiol 41:832-838

25. Brady Z, Cain TM, Johnston PN (2011) Paediatric CT imaging trends in Australia. J Med Imaging Radiat Oncol 55:132-142

26. Miglioretti DL, Johnson E, Williams A et al (2013) The use of computed tomography in pediatrics and the associated radiation exposure and estimated cancer risk. JAMA Pediatr 167:700-707

27. Meulepas JM, Ronckers CM, Smets AMJB et al (2014) Leukemia and brain tumors among children after radiation exposure from CT scans: design and methodological opportunities of the Dutch Pediatric CT Study. Eur J Epidemiol 29:293-301

28. Hansen J, Jurik AG (2009) Analysis of current practice of CT examinations. Acta Oncol 48:295-301 